\title{
MAGNETICA, une expo attirante
}

Hélène Fischer (helene.fischer@univ-lorraine.fr)

Institut Jean Lamour (UMR7198 CNRS / Université de Lorraine), Campus ARTEM

2 allée André Guinier, BP 50840, 54011 Nancy Cedex

L'exposition itinérante tout public "MAGNETICA, une expo attirante » a pour objectif de mettre à mal les croyances liées au magnétisme. D'esthétique cristalline

et attractive, elle présente

une approche scientifique expérimentale du magnétisme, afin d'en expliquer les principes

et les effets à l'origine

de nombreuses applications

omniprésentes dans nos vies,

et à l'origine de recherches

actuelles.

L'idée directrice est de partager

connaissance et culture sur tout

le territoire, d'attiser le goût des

sciences chez le grand public,

d'intéresser plus particulièrement

les jeunes aux métiers

scientifiques, à la recherche

et à l'innovation, d'initier leur

esprit pour les aider à se projeter

dans l'avenir.

\section{Le contexte de création de MAGNETICA}

"MAGNETICA, une expo attirante » est une exposition grand public sur le magnétisme, produite dans le cadre du projet Escales des Sciences porté par l'Université de Lorraine (encadré 1). Elle est le fruit d'une démarche atypique de médiation scientifique mêlant scientifiques et designers, pour offrir au public la découverte du magnétisme au fil de nombreuses expériences à tester par soi-même, depuis les aimants du quotidien jusqu'à des résultats de recherches concernant le magnétisme. En portant ce projet, l'équipe «nano-magnétisme et électronique de spin " de l'Institut Jean Lamour (IJL) (encadré 2) a affirmé sa volonté de faire découvrir les sciences à un large public, affichant ainsi son implication dans la diffusion de la culture et de l'information scientifiques. L'objectif est multiple : éveiller et former à la science et à la démarche scientifique, expliquer et débattre des avancées et des enjeux de la recherche avec les citoyens, et même peut-être susciter des vocations scientifiques.

L'évolution de la société actuelle, en particulier la façon d'apprendre des plus jeunes, nous amène à réfléchir et à diversifier nos façons de vulgariser et partager la science avec le grand public. De nouveaux formats adaptés à un auditoire toujours plus exigeant, habitué à plus d'interactivité, sont à développer. Mais, innover, créer des actions de qualité, capables d'attirer une audience de plus en plus sollicitée, est complexe. La présence de chercheurs dans cette démarche de médiation est irremplaçable : d'une part, ils sont un gage de qualité quant au contenu scientifique ; d'autre part, ils peuvent parler des recherches récentes et ancrer la science dans la modernité. Comme ils sont les acteurs de la recherche, ils peuvent témoigner de cette pratique, de leur vécu, de leur passion, de leurs questionnements. D'où l'intérêt de ce projet, mené par des chercheurs de l'Institut Jean Lamour : le point de vue est celui du physicien issu de la communauté du magnétisme, enrichi par des collaborations interdisciplinaires avec des chercheurs d'autres communautés.

La présence de compétences complémentaires non scientifiques se révèle nécessaire aussi au développement d'une médiation scientifique adaptée à la société actuelle. La récente implantation de l'Institut Jean Lamour au sein d'un nouveau campus baptisé ARTEM, acronyme issu des mots ARt, TEchnologie et Management (encadré 3), offre la possibilité d'explorer une nouvelle déclinaison de la médiation scientifique en exploitant la richesse des synergies émanant du rapprochement de l'art et des sciences dures. L'originalité de l'action menée avec MAGNETICA est d'inscrire la démarche de transmission des connaissances scientifiques dans une scénographie issue d'un travail mené avec une école d'art et de design. Finalement, le but ultime est d'élaborer un contenu, intrinsèquement de qualité car produit par des chercheurs, immergé dans un contenant également de qualité, car imaginé par des artistes.

Ainsi, "MAGNETICA, une expo attirante » est à la fois le fruit d'une immersion dans la science en création au sein d'un laboratoire de recherche, d'un goût pour le partage des connaissances, du désir d'associer le grand public à l'émerveillement soulevé par la découverte scientifique, et des synergies émergeant de l'interdisciplinarité du campus ARTEM. La collaboration étroite entre physiciens et jeunes étudiants originaires des deux écoles du campus 
ARTEM (Mines Nancy et l'école d'art et de design ENSAD) a permis de concevoir et développer de nouveaux supports, incluant les codes actuels de communication, illustrant la démarche d'investigation du scientifique, des maquettes à la fois pédagogiques et esthétiques, pour montrer la physique différemment et permettre au grand public d'approcher une démarche scientifique. Finalement, le fil conducteur suivi rejoint la vision de Jean Perrin qui, à la création du Palais de la découverte, voulait proposer au public un lieu pour "voir la science en train de se faire ", un lieu esthétique où le public expérimente la démarche d'investigation pour créer la science.

\section{Une approche scientifique expérimentale du magnétisme}

"MAGNETICA, une expo attirante " propose au public de se laisser porter par une histoire, une histoire soutenue par l'expérimentation, construite selon un fil directeur logique, qui amène chaque table à appeler la suivante. Cette construction revisite les fondamentaux du magnétisme et dessine leurs imbrications, aidant ainsi à comprendre ce qu'est le magnétisme. Pour l'accroche, cette histoire débute par ce que tout le monde connait, l'aimant que l'on colle au frigo, puis explique pas à pas les aspects fondamentaux du magnétisme en les mettant en évidence grâce à des maquettes expérimentales, certaines très pédagogiques et esthétiques, d'autres illustrant une application du quotidien directement issue du principe fondamental étudié. L'explication scientifique du phénomène observable figure sur un cartel situé à côté de chaque expérience à réaliser. Petit à

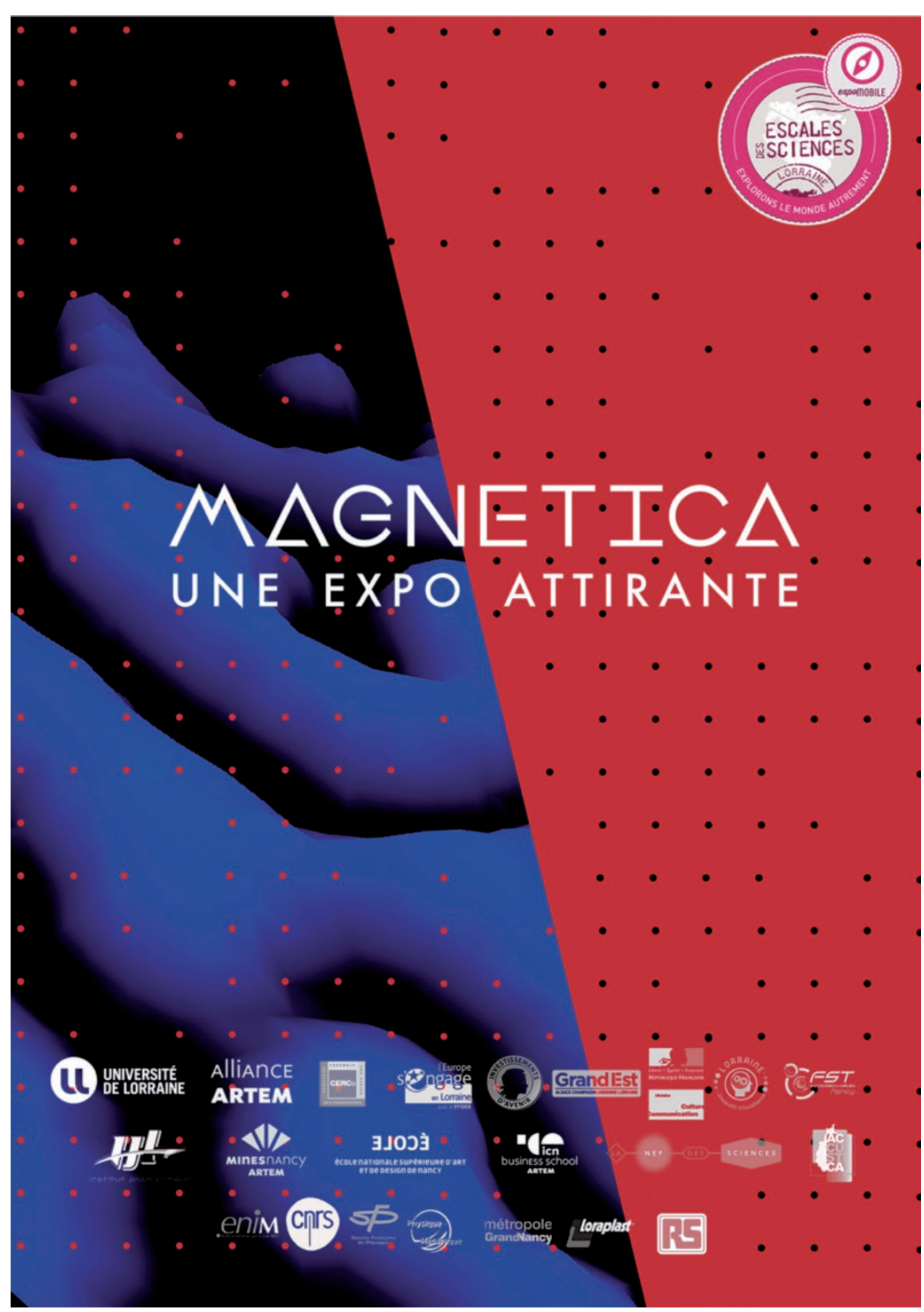

Le projet Escales des Sciences

Encadré 1

Porté par l'Université de Lorraine, ce projet est une démarche qui vise à diffuser les cultures scientifique, technique et industrielle sur le territoire du Grand Est, et maintenant aussi sur le territoire national. Née du programme d'Investissement d'avenir CERCo (Construisons Ensemble une Région de la Connaissance), Escales des Sciences est une initiative inspirée par la société et pour la société. Au cœur du projet : la volonté de réduire les disparités principalement observées entre les zones urbaines et rurales, et de promouvoir une égalité d'accès à la culture ; l'envie de faire découvrir autrement et au plus grand nombre, le monde d'aujourd'hui et d'initier à celui de demain, et de donner à tous les citoyens, petits et grands, davantage d'occasions d'explorer les sciences actuelles et de s'interroger sur la manière dont elles imprègnent notre quotidien et construisent notre futur. 
petit, par l'expérimentation et l'analyse de la signification physique de l'expérience effectuée, cette exposition va jusqu'à apporter au public les connaissances nécessaires à la compréhension de certains résultats de recherches menées dans le domaine du magnétisme à l'Institut Jean Lamour (IJL). Elle permet également de rendre compte de la démarche d'investigation du chercheur.

L'exposition est structurée autour de cinq îlots thématiques, qui répondent à l'ensemble des questions : où trouve-t-on du magnétisme? Comment expliquer ce phénomène ? Quelles en sont les applications ? Quel rôle joue le magnétisme dans un ordinateur ? Et quelles recherches mène-t-on sur le magnétisme à l'IJL ?

Chacun de ces îlots comporte de nombreux dispositifs expérimentaux que les visiteurs peuvent manipuler. Tous ces dispositifs sont uniques mais respectent le même design : leur conception technique et leur réalisation

\section{L'Institut Jean Lamour (IJL)}

L'IJL est un laboratoire nancéen de recherche fondamentale et appliquée en science et ingénierie des matériaux et des procédés. Unité mixte de l'Université de Lorraine et du CNRS, I'IJL rassemble 550 personnes et couvre de nombreuses thématiques. Ses priorités scientifiques sont définies par six axes de recherche transverses : plasmas chauds et froids - fusion thermonucléaire ; matériaux artificiels nanostructurés ; interfaces avancées pour l'énergie ; métallurgie ; théorie, modélisations et simulations ; matériaux et vivant.

Le campus ARTEM

Encadré 3

ARTEM, comme ARt, TEchnologie et Management, est un campus interdisciplinaire d'un nouveau genre : il puise tout son sens de l'étymologie du terme latin ars, artis qui recouvrait un champ très vaste allant de la culture au talent, de la science au métier, de la connaissance à la production. ARTEM réunit l'École Nationale Supérieure d'Art et de Design de Nancy (I'ENSAD), Mines Nancy, et I'ICN Business School. Il est construit en écho au mouvement Art nouveau que fut (à la fin du $19^{\mathrm{e}}$ et au début du $20^{\mathrm{e}}$ siècle) l'École de Nancy, dont le but était de réconcilier art et industrie. également la présentation du fleuron de l'Institut Jean Lamour, le TUBE (comme inTerconnexion under Ultra-high vacuum of ChamBers for Elaboration and Characterization for Novel Material) : il s'agit d'un outil unique au monde, développé sur le site d'ARTEM par le centre de compétence en Dépôt et Analyse sous Ultravide de nanoMatériaux (acronyme DAUM, clin d'œil à la célèbre cristallerie nancéenne). Cet outil phare combine un large éventail de techniques de synthèses et de caractérisations de films minces, et permet de développer des projets scientifiques multimatériaux et multi-analyses dans le domaine des nanomatériaux. L'exposition permet de partager cet équipement unique avec le grand public au travers de maquettes ou films, et d'en expliquer le sens et l'intérêt pour la réalisation de matériaux nouveaux, aux propriétés nouvelles, comme par exemple les empilements à magnétorésistance géante. L'exposition présente également, toujours sous forme de maquettes et tablettes, les principes de la lithographie permettant de structurer latéralement les films élaborés, ainsi que certaines expériences menées pour caractériser structuralement ou magnétiquement les nano-objets créés. Pour être complet et ne pas seulement rendre compte des activités en recherche fondamentale, l'exposition illustre aussi deux exemples de recherche appliquée menée dans le laboratoire (thèses cofinancées par un industriel) dans le domaine du magnétisme.

L'exposition contient encore un sixième îlot intitulé "Magnétisme à ARTEM : un jeu d'enfants ! ": il permet de balayer de façon ludique l'ensemble des phénomènes illustrés dans l'exposition à travers un nouveau module du célèbre jeu Minecraft, un Minecraft magnétique, développé par des élèves de Mines Nancy.

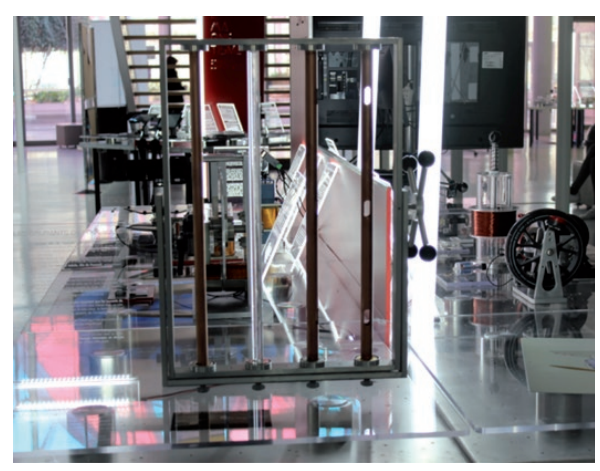

Vue d'ensemble de l'îlot III, intitulé «MAGNÉTISME : pour quoi faire? »

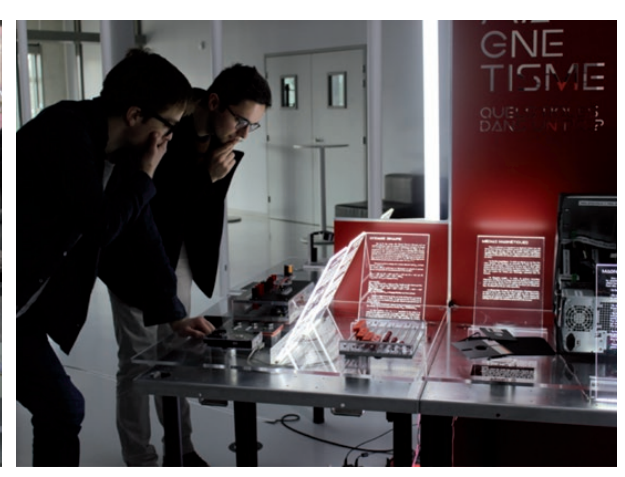

Vue d'ensemble de l'îlot IV, intitulé "MAGNÉTISME : quels rôles dans un PC ?"

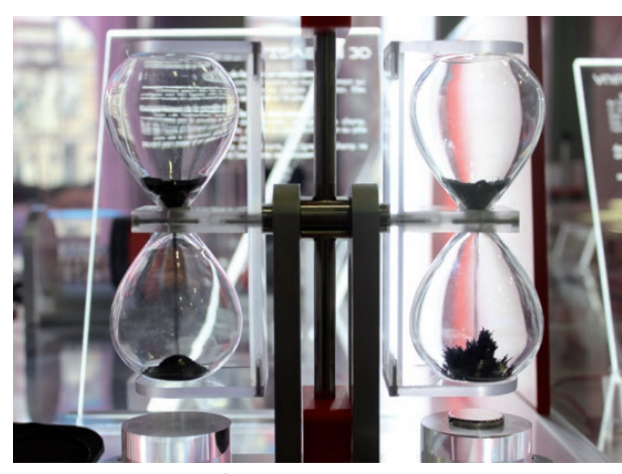

Le sablier magnétique. Dans le sablier de droite placé au-dessus d'un aimant, la poudre de fer s'aligne sur les lignes de champ. 
Enfin, un totem central aux six îlots rassemble des informations sur les origines étymologique et historique du magnétisme, quelques réflexions philosophiques au sujet des croyances occultes liées aux phénomènes magnétiques, et les différentes étapes du développement d'une approche scientifique du magnétisme jusqu'aux deux prix Nobel français sur le sujet, Louis Néel et Albert Fert.

Depuisle printemps 2017, «MAGNETICA, une expo attirante " est également intégrée au projet IMPACT NanoMaterial for Sensors (N4S) de l'I-SITE Lorraine Université d'Excellence, qui s'inscrit dans le défi "maitrise de l'ensemble de la chaîne des matériaux ". N4S est un projet tourné vers l'excellence de la recherche scientifique, le transfert technologique, et également la CSTI dans le domaine des nanomatériaux structurés. L'axe du projet N4S consacré à la médiation scientifique et organisé en un centre de compétences, vise à partager avec le grand public les avancées scientifiques engendrées par ce projet N4S. "MAGNETICA, une expo attirante " s'inscrit tout naturellement dans cette thématique : l'exposition apporte toutes les connaissances nécessaires pour présenter et expliquer au grand public les innovations apportées par IMPACT N4S dans le domaine du magnétisme.

\section{Une exposition liant la science à l'art et aux acteurs de la recherche}

Le long travail mené au sein d'un atelier ARTEM, impliquant entre autres des étudiants de l'École d'Art et de Design de Nancy (l'ENSAD) confereà $M A G N E T I C A$ une esthétique attractive et appréciée du public. Le design de l'exposition trouve sa force dans la symbolique de la transparence, illustrant l'objectif de la démarche de vulgarisation scientifique, qui est de rendre la science accessible, donc transparente. Ainsi, l'exposition est cristalline, toutes les expériences sont montées sur panneaux transparents qui semblent léviter au-dessus du vide, marquant ainsi l'intemporalité du savoir. Tous les éléments techniques sont visibles. Les cartels explicatifs sont eux aussi transparents, donc peu lisibles en l'état. Illuminés, leur sens jaillit et illustre la lumière de la connaissance.

"MAGNETICA, une expo attirante " lie aussi la science aux chercheurs et à tous les acteurs de la recherche : en effet, de petites cartes symboliques, à leur image, sont disséminées dans l'exposition. Elles sont le moyen de découvrir des visages d'acteurs de la recherche, soit impliqués dans le projet, soit impliqués dans des recherches en rapport avec l'exposition. Chaque carte, déclinée dans le design de l'exposition, associe un prénom, une photo, et quelques mots pour définir soit la fonction de la personne, soit son champ de recherche, soit sa contribution à l'exposition. L'idée est de présenter les différents métiers associés à la recherche, d'illustrer la présence des étudiants impliqués dans MAGNETICA, de démystifier le chercheur en le présentant comme un homme ou une femme abordable, normal(e), jeune ou moins jeune, et de montrer que les fondamentaux exposés dans MAGNETICA ne sont pas obsolètes ou dépassés : les chercheurs sont présents pour témoigner de leur utilité quotidienne dans les recherches actuelles.

\section{Une exposition conçue pour l'itinérance}

"MAGNETICA, une expo attirante" a été inaugurée fin juin 2017. Elle a été exposée en octobre à la Faculté des Sciences et Technologies de Vandœuvre, pendant la semaine de la Fête de la science où elle a rencontré un franc succès. Elle a été ensuite exposée d'octobre 2017 à février 2018 à Mines Nancy, puis à la mairie d'Essey-lès-Nancy. Elle fait l'objet de nombreuses demandes de visites guidées individuelles et de visites scolaires de niveau lycée. Elle a donné naissance à des articles dans la presse locale, et à deux reportages TV : http://videos.univ-lorraine. fr/index.php?act $=$ view\&id_col $=105$

MAGNETICA est ouverte à la location et destinée à l'itinérance sur le territoire. Elle peut être réservée pour une location auprès du service Escales des Sciences de l'Université de Lorraine. La surface minimale nécessaire pour l'accueillir est de 200 à $250 \mathrm{~m}^{2}$. L'exposition est accompagnée de supports pédagogiques écrits et audiovisuels, destinés soit à des médiateurs, soit au public. Un dépliant de visite-jeu pour enfants a aussi été développé. Un site web est en préparation.

\section{Vous pouvez également retrouver MAGNETICA \\ en téléchargeant la vidéo}

https://drive. google. .com/open?id=1i4MnvP

Q1M0V23XOMLkmUZ088yd_PfuKS

et à l'adresse

www.facebook.com/

MagneticaUneExpoAttirante/.

\section{Informations sur l'exposition : Hélène Fischer - helene.fischer@univ-lorraine.fr Réservation de l'exposition : Fanny Wagner - escales-des-sciences@univ-lorraine.fr}

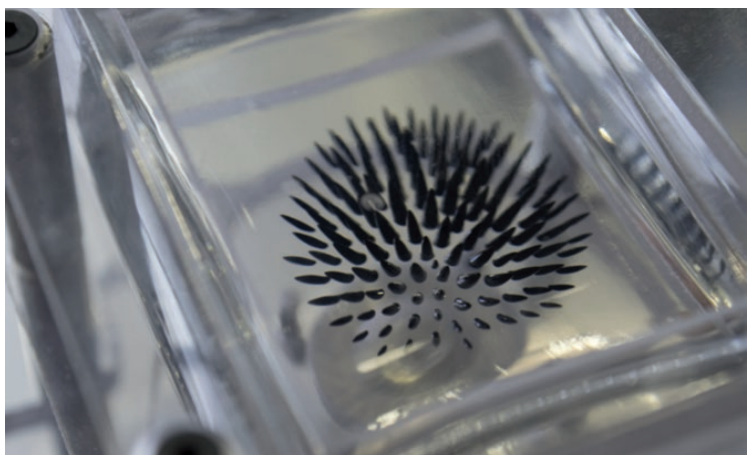

Le jeu du hérisson. La montée et la descente d'un aimant placé sous un flacon contenant une goutte de ferrofluide, permet de faire apparaitre ces figures en hérisson.

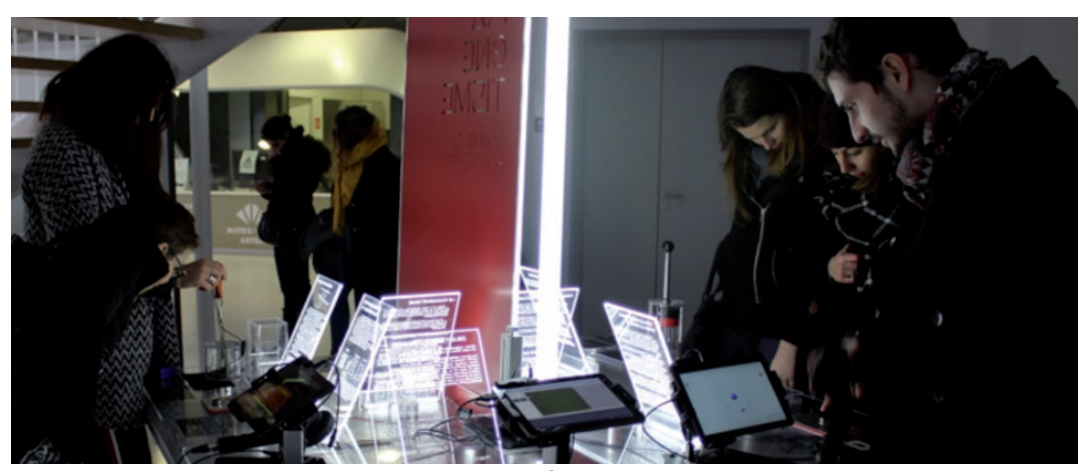

Vue d'ensemble de l'îlot II, intitulé « MAGNÉTISME : comment l'expliquer ? » 